Journal of

Synchrotron

Radiation

ISSN 0909-0495

Editors: Å. Kvick, D. M. Mills and T. Ohta

\title{
The new HMI beamline MAGS: an instrument for hard X-ray diffraction
} at BESSY

\section{Esther Dudzik, Ralf Feyerherm, Wolfgang Diete, Riccardo Signorato and} Christopher Zilkens

Copyright $($ International Union of Crystallography

Author(s) of this paper may load this reprint on their own web site provided that this cover page is retained. Republication of this article or its storage in electronic databases or the like is not permitted without prior permission in writing from the IUCr. 
Journal of

Synchrotron

Radiation

ISSN 0909-0495

Received 26 April 2006

Accepted 15 August 2006

\section{The new HMI beamline MAGS: an instrument for hard X-ray diffraction at BESSY}

\author{
Esther Dudzik, ${ }^{a *}$ Ralf Feyerherm, ${ }^{a}$ Wolfgang Diete, ${ }^{b}$ Riccardo Signorato ${ }^{b}$ and \\ Christopher Zilkens ${ }^{b}$
}

a Hahn-Meitner-Institut Berlin, Albert-Einstein-Strasse 15, 12489 Berlin, Germany, and ${ }^{\mathbf{b}} \mathrm{ACCEL}$
Instruments GmbH, Bergisch Gladbach, Germany. E-mail: dudzik@hmi.de

The Hahn-Meitner-Institute Berlin is operating the new hard X-ray diffraction beamline MAGS at the Berlin synchrotron radiation source BESSY. The beamline is intended to complement the existing neutron instrumentation at the Berlin Neutron Scattering Centre. The new beamline uses a $7 \mathrm{~T}$ multipole wiggler to produce photon fluxes in the $10^{11}-10^{12}$ photons s$^{-1}(100 \mathrm{~mA})^{-1}(0.1 \%$ bandwidth $)^{-1}$ range at energies from 4 to $30 \mathrm{keV}$ at the experiment. It has active bendable optics to provide flexible horizontal and vertical focusing and to compensate the large heat load from the wiggler source. The experimental endstation consists of a six-circle Huber diffractometer which can be used with an additional (polarization) analyser and different sample environments. The beamline is intended for single-crystal diffraction and resonant magnetic scattering experiments for the study of ordering phenomena, phase transitions and materials science.

Keywords: beamline; X-ray diffraction; resonant scattering; magnetic scattering; ordering phenomena.
(C) 2006 International Union of Crystallography Printed in Great Britain - all rights reserved they had to be designed and built simultaneously. The two experiments have separate radiation safety hutches and are operated fully independently.

The basic beamline design and specifications were supplied by the HMI, while the detailed design, construction and installation of the beamline was carried out by ACCEL Instruments $\mathrm{GmhH}$, who designed the beamline up to the first valve in the experimental hutch. The most challenging part of the design was the handling of the high heat load from the multipole wiggler source.

First experimental results prove that the beamline MAGS is a versatile tool for structural investigations in a variety of different research areas ranging from basic solid-state physics to applications in materials science.

\section{Beamline description}

\subsection{The wiggler source}

The $7 \mathrm{~T}$ multipole wiggler has been described elsewhere (Berger et al., 2002), so only a brief description is given here. It was designed jointly by BESSY and the HMI, and built by the Budker Institute in Novosibirsk (Russia). It is a superconducting $7 \mathrm{~T}$ wiggler with 13 full poles and $3 / 4$ and $1 / 4$ end poles with a period length of $140 \mathrm{~mm}$. The wiggler source consists of an array of point sources separated horizontally by $1.22 \mathrm{~mm}$ and by half the period length along the beam direction, which originate at the 'turning points' of the 'wiggling' 
electron beam path. Seen from the beamline perspective, the source consists of two spots, each made up of one row of the individual wiggler source spots. Since the MAGS beamline is built $1 \mathrm{mrad}$ off-axis, the two rows appear slightly staggered, which effectively smears out the two source spots. At the experiment, the beam is about $92 \%$ linearly polarized in the orbit plane with the vertical white beam slits fully open.

The vacuum vessels downstream of the wiggler and the front-end were designed by BESSY staff to handle the maximum power load of $55 \mathrm{~kW}$ at $1.9 \mathrm{GeV}$ ring energy and $500 \mathrm{~mA}$ ring current. Under the current BESSY operating conditions (1.7 GeV ring energy, $250 \mathrm{~mA}$ injection current) the actual heat load is $22 \mathrm{~kW}$. The wiggler critical energy is $13.5 \mathrm{keV}$ at $1.7 \mathrm{GeV}$ and $16.9 \mathrm{keV}$ at $1.9 \mathrm{GeV}$.

\subsection{Beamline optics}

Fig. 1 shows the overall layout of the beamline optics. The front-end and a fixed water-cooled copper white-beam mask cut a $3 \mathrm{mrad} \times 0.3 \mathrm{mrad}(\mathrm{h} \times \mathrm{v})$ section out of the wiggler beam, which corresponds to a maximum heat load of $2000 \mathrm{~W}$ (at $1.9 \mathrm{GeV}$ and $500 \mathrm{~mA}$ ). The beam then passes through a $400 \mu \mathrm{m}$-thick Be window with a $100 \mu \mathrm{m}$ carbon pre-filter which is used to separate front-end and beamline vacuum. The $\mathrm{C}$ pre-filter reduces the heat load on the $\mathrm{Be}$ window from $220 \mathrm{~W}$ to about $20 \mathrm{~W}$.

The next components are vertical and horizontal whitebeam slits at $17 \mathrm{~m}$ from the source. The slits are water-cooled and designed to withstand the full heat load when closed. They are followed by four graded absorbers that can be used to reduce the thermal load on the downstream optical components, particularly when the mirrors are retracted from the beam at high photon energies. Each absorber in turn removes approximately $50 \%$ of its incident load, with a remaining heat load of just $70 \mathrm{~W}$ when all filters are in.

The beam is vertically collimated by the first mirror at $20.5 \mathrm{~m}$ from the source. A water-cooled white-beam aperture was placed between collimating mirror and monochromator to prevent stray light from the mirror from hitting the monochromator mechanics and chamber walls. The fixed-exit double-crystal monochromator is positioned at $23 \mathrm{~m}$, and the beam is vertically refocused by the second mirror at $25.5 \mathrm{~m}$. The experiment is at $36.5 \mathrm{~m}$ from the source. At photon energies above $15 \mathrm{keV}$ both mirrors are retracted from the beam. In this geometry the beam is still horizontally focused by the sagittal bender, and collimated vertically by the slits.

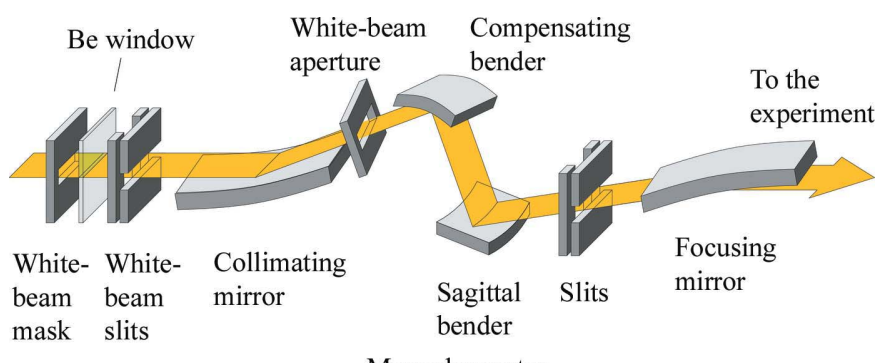

Figure 1

Monochromator
The changeover between the mirrorless mode and the vertically focused mode takes about $30 \mathrm{~min}$.

\subsection{Mirrors}

The mirrors and the mirror benders were built by SESO, France. Both mirror substrates are Si single crystals, $1200 \mathrm{~mm}$ long, $200 \mathrm{~mm}$ wide and $50 \mathrm{~mm}$ thick, with the reflecting surfaces polished to $3 \AA$ r.m.s. roughness. The mirrors have two tracks: one is coated with $\mathrm{Rh}$ and the other is the uncoated Si substrate. For both mirrors the pitch angle can be varied between 0 and $5 \mathrm{mrad}$. The variable pitch and the different coatings are used to match the mirror reflectivity cut-off to the photon energy for harmonic rejection.

The mirrors can be retracted from the beam to allow work at photon energies above $15 \mathrm{keV}$. Behind each mirror a fluorescent screen can be moved into the beam to check the beam position. The mirror vessel vacuum is about $5 \times 10^{-7} \mathrm{~Pa}$ with the beam on. There are no foils separating the mirror vacuum from the monochromator vacuum (in the $10^{-6} \mathrm{~Pa}$ range, depending on heat load); instead the two are separated by a differentially pumped absorber mask.

The collimating mirror at $20.5 \mathrm{~m}$ from the source reflects upwards. It is mounted on a four-cylinder SESO bender and has a variable bending radius to adapt to changes in pitch and heat load, with bending radii between 40 and $4 \mathrm{~km}$. The mirror is indirectly water-cooled, with cooling pipes running through grooves cut into the mirror substrate. A eutectic liquid (Galinstan) serves as heat conductor between the substrate and the cooling pipe. The cooling was designed for heat loads up to $2000 \mathrm{~W}$.

The refocusing mirror at $25.5 \mathrm{~m}$ is mounted on a SESO U-bender. It reflects downwards, with a variable bending radius between $\infty$ and $2.3 \mathrm{~km}$. This mirror needs no cooling.

\subsection{Monochromator}

The fixed-exit double-crystal monochromator at $23 \mathrm{~m}$ from the source covers the energy range from 4 to $30 \mathrm{keV}$ with a pair of $\mathrm{Si}(111)$ crystals, and can reach photon energies up to approximately $70 \mathrm{keV}$ using the $\mathrm{Si}(333)$ reflection. With the collimating mirror in the beam, the energy resolution at the $\mathrm{Cu} K$ edge is $2 \mathrm{eV}$; without mirrors the energy resolution is limited by the vertical beam divergence of $0.3 \mathrm{mrad}$.

The monochromator crystals are mounted on a goniometer, with the first crystal surface at the centre of rotation. The goniometer can be moved vertically by $50 \mathrm{~mm}$ to adjust to the changing first-mirror pitch. The first crystal is indirectly watercooled, with the cooling designed for heat loads up to $600 \mathrm{~W}$. The cooling is combined with a counterbending mechanism to compensate the thermal bump from the wiggler beam (Zaeper et al., 2002). This bender was built by ACCEL in collaboration with the University of Wuppertal, Germany. When the mirrors are in the beam the maximum heat load is about $300 \mathrm{~W}$. The first-crystal cooling is so efficient that in this case the compensating bender is not necessary. Without the mirrors, higher heat loads can easily be reached, and here the operation of the compensating bender can be seen in Fig. 2. At 


\section{research papers}
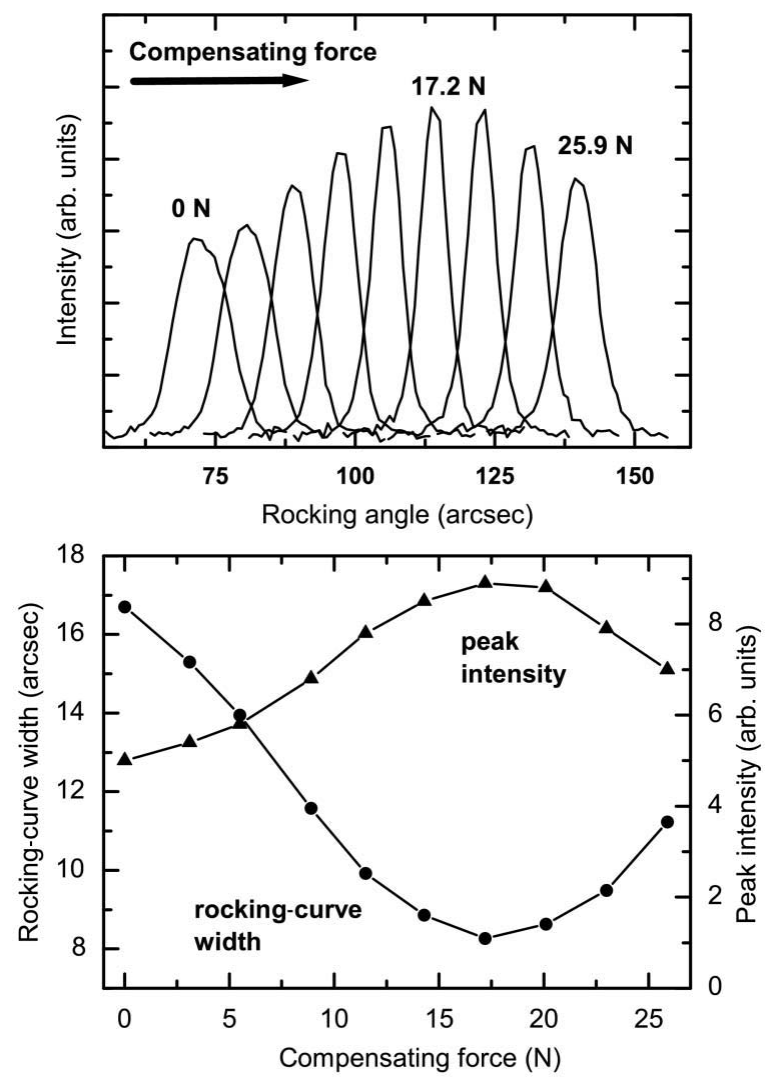

Figure 2

Top: set of rocking curves at $800 \mathrm{~W}$ heat load (offset for clarity). Bottom: corresponding reduction of the rocking-curve width/increase of peak intensity when the counterbender is used.

$9 \mathrm{keV}$ no mirrors were used. With the white-beam slits fully open at a high ring current, a heat load of approximately $800 \mathrm{~W}$ falls on the first monochromator crystal. The uncompensated rocking curve is broadened by the thermal distortion of the crystal to twice the theoretical width of 8.3 arcsec. When force is applied to the bender, the rocking curve narrows down to the theoretical rocking-curve width with a corresponding increase in intensity, shown in Fig. 2. Further details about the compensating bender can be found in a separate publication (Dudzik et al., 2006).

The second crystal is mounted on an ESRF-type sagittal bender (Freund et al., 1998) with a variable bending radius between 1 and $80 \mathrm{~m}$. It provides 2:1 horizontal focusing at all photon energies. A vertical crystal translation of range $50 \mathrm{~mm}$ and a horizontal translation of $200 \mathrm{~mm}$ provide a fixed beam offset of about 25-30 mm (this can be chosen to some extent). There is a coarse- and a fine-pitch adjustment for the second crystal, along with a roll and a yaw movement. Fig. 3 shows the possible focus settings of the beamline: $(a)$ completely unfocused beam, $(b)$ horizontal focusing (the two stripes are the image of the two source spots in the wiggler), $(c)$ vertical focusing, and $(d)$ both horizontal and vertical focusing. The focus is made up of two spots of size $\sim 0.5 \mathrm{~mm} \times 0.4 \mathrm{~mm}(\mathrm{~h} \times$ $\mathrm{v})$, separated horizontally by $1 \mathrm{~mm}$. All figures are to the same scale indicated by the white $1 \mathrm{~mm}^{2}$ square in $(d)$. In practice the split focus is not too problematic. Very small crystals are
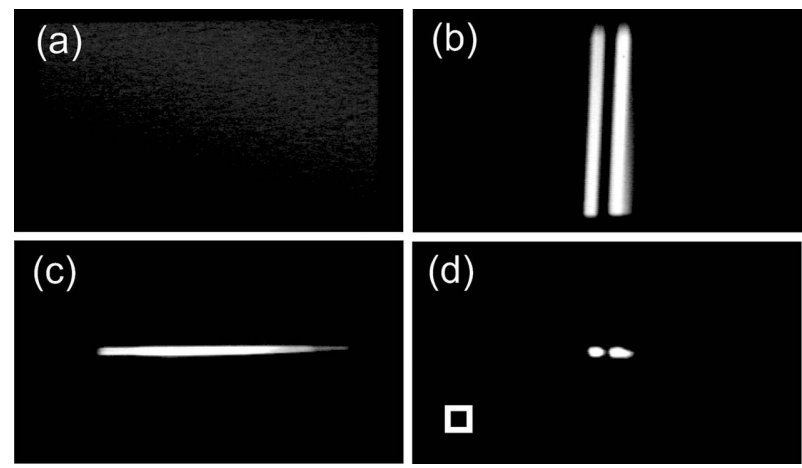

Figure 3

Possible focus settings of the beamline. (a) Unfocused beam; (b) horizontal focusing; $(c)$ vertical focusing; $(d)$ both horizontally and vertically focused beam. The white square has a size of $1 \mathrm{~mm} \times 1 \mathrm{~mm}$ to indicate the scale. The two spots in the horizontal focus are images of the wiggler source spots.

positioned in one of the two spots; larger samples receive the full beam. Because of the horizontal beam divergence (up to $6 \mathrm{mrad}$, depending on the horizontal white-beam slit size), the two focus spots are not resolved at the detector position (about $0.6 \mathrm{~m}$ from the sample). They would only become visible if a high-resolution experiment with reduced horizontal beam divergence and small detector slits was to be carried out in the horizontal scattering geometry; in this case one of the focus spots would have to be masked.

Behind the monochromator there is a second set of slits, as well as an intensity monitor $\left(I_{0}\right)$ which consists of a scattering foil and a photodiode. $I_{0}$ can be used as input into a monochromator stabilization unit (MOSTAB) (Meß et al., 1998). In practice it turns out that the MOSTAB is not really necessary. Even with heat loads changing considerably between injections (by a factor of two), the centre of the second-crystal rocking curve remains stable within 1 arcsec at fixed photon energies and during energy scans over ranges of a few hundred $\mathrm{eV}$.

\section{Experimental set-up}

The last section of the beamline (designed by HMI staff) is inside the experimental hutch. It contains a set of graded attenuators followed by horizontal and vertical slits and another $I_{0}$ (again using a scattering foil and a photodiode). All these components are inside the beamline UHV, in order to keep the number of windows in the beamline at a minimum to allow work at photon energies down to $3.5 \mathrm{keV}$. The Be window at the end of the beamline is $250 \mu \mathrm{m}$ thick.

The diffractometer is a six + three-circle Huber diffractometer with a standard 512 Eulerian cradle. The diffractometer can be used both in the vertical and the horizontal scattering geometry. It has an optional polarization/energy analyser with a full set of analyser crystals in the energy range from $3.5 \mathrm{keV}$ to $20 \mathrm{keV}$.

The diffracted beam then passes through two sets of slits which make up a collimation path. The first slits were made by JJ-XRAY; the second set of slits are part of the Huber 


\section{research papers}

analyser. Two detector systems are available. A liquidnitrogen-cooled Canberra low-energy Ge diode detector with a Canberra digital signal processor and a multi-channel analyser provides an easy way of separating fluorescence, higher-order light etc. An Oxford Danfysik Cyberstar scintillation detector serves as a robust everyday detection system, and is also small enough to be used with the analyser.

Two closed-cycle Displex-based cryostats are available for sample temperatures from 6 to $800 \mathrm{~K}$ and from 4 to $300 \mathrm{~K}$, respectively. Another closed-cycle cryostat for temperatures down to $1.5 \mathrm{~K}$ will be available in the near future; a superconducting 5.5 $\mathrm{T}$ magnet is under development.

Beamline and experiment are controlled by two separate Linux PCs with spec (Swislow, 1996) software from Certified Scientific Software. On the beamline control PC, spec runs in server mode so that beamline motors can be accessed from the spec program running on the experimental control PC. Both spec programs read in EPICS control variables (ring current, wiggler field etc.) from the BESSY control system. The experiment spec controls the end of the beamline, the diffractometer and sample environment, as well as the detector system via GPIB and VME interfaces.

\section{First results}

By now, a range of solid-state physics and materials science experiments have been performed on MAGS. Fig. 4 (top)
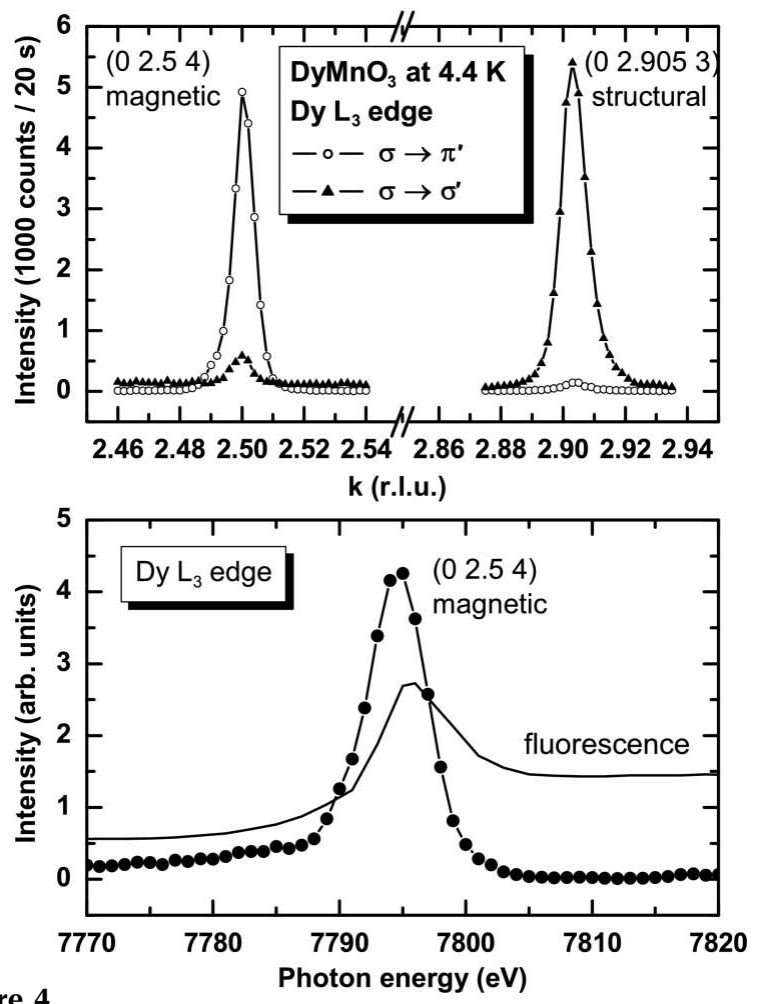

Figure 4

Photon energy $(\mathrm{eV})$

Top: polarization analysis of a magnetic and a structural superstructure peak in $\mathrm{DyMnO}_{3}$. Bottom: energy dependence of the magnetic peak intensity at the Dy $L_{3}$ edge. The magnetic peak is strongly enhanced in resonance (the position of the edge was determined by measuring the energy dependence of the diffuse fluorescence background away from the Bragg peaks). shows the results of polarization analysis in resonant magnetic scattering, performed on a magnetic and a structural superlattice peak in orthorhombic $\mathrm{DyMnO}_{3}$ (Feyerherm et al., 2006). Polarization analysis makes it possible to distinguish between magnetic and structural contributions to the scattered signal (Blume \& Gibbs, 1988; Hill \& McMorrow, 1996). The measurements shown here were made close to the Dy $L_{3}$ edge at $7794 \mathrm{eV}$, with a HOPG polarization analyser using the graphite (006) reflection with $0.35^{\circ}$ mosaic width. On resonance and below the transition temperature of $5 \mathrm{~K}$, magnetic superstructure peaks appear at positions $(0 \mathrm{k} / 2 \mathrm{l})$ in reciprocal space, due to the antiferromagnetic ordering of the rare-earth ions in the crystal. Polarization analysis confirms the magnetic origin of these peaks; here scattering is predominantly from the in-plane linear polarization $(\sigma)$ into the vertical polarization $\left(\pi^{\prime}\right)$, while structural scattering of a second incommensurate superstructure peak leaves the original $\sigma$-polarization unchanged. Fig. 4 (bottom) shows the energy dependence of the magnetic diffraction peak intensity at the Dy $L_{3}$ edge. The magnetic peak is strongly enhanced (by a factor of $\sim 20$ ) on resonance, with its intensity almost zero above the resonance. These results demonstrate that the beamline MAGS is well suited for the demanding resonant magnetic scattering technique which generally is intensity limited, although in this context it has to be noted that the measurements had to be carried out with a reduction of the incident beam intensity by a factor of 0.33 in order to prevent sample heating.

Fig. 5 shows an energy scan across the Ho $K$ edge at $55.62 \mathrm{keV}$, measured in transmission on a $100 \mu \mathrm{m}$-thick Ho foil. To reach this high photon energy the monochromator was set to use the $\mathrm{Si}(333)$ reflection. This was a test measurement to see if the monochromator was capable of handling the narrow rocking-curve width of 0.2 arcsec without going out of alignment during an energy scan. Although the statistics are not good (this was a rapid scan with a photodiode as detector), the energy scan worked quite well and reproducibly. Thus, we are confident that experiments at MAGS can be carried out up to a photon energy of about $70 \mathrm{keV}$.

Fig. 6 shows a result from a materials science experiment conducted at $30 \mathrm{keV}$ in the mirrorless mode. Here residual

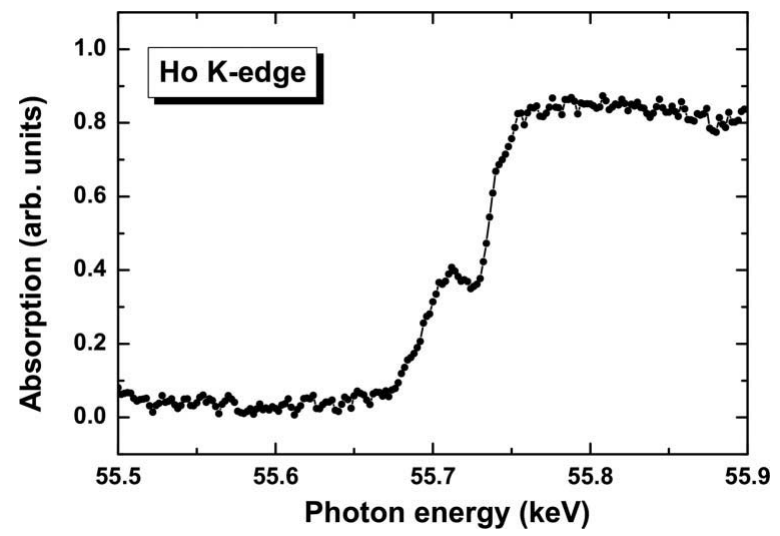

Figure 5

The Ho $K$ edge at $55.62 \mathrm{keV}$, measured in transmission through a $100 \mu \mathrm{m}$ Ho foil. 

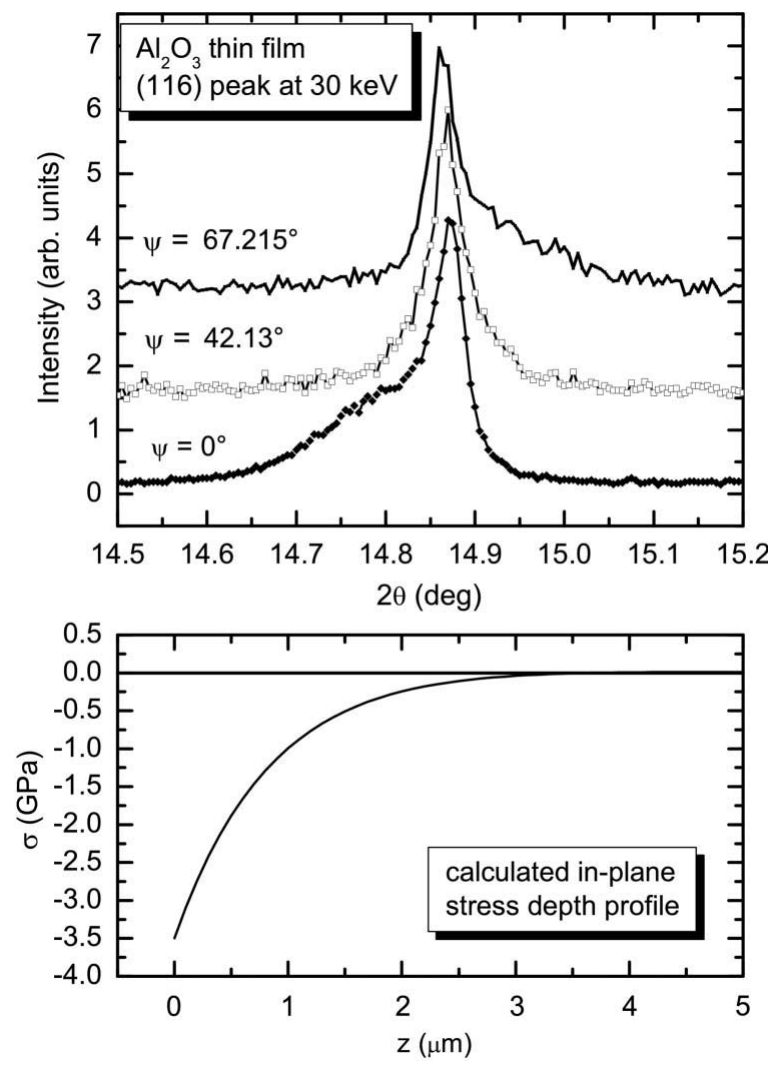

Figure 6

Top: the (116) powder diffraction peak of a $6 \mu \mathrm{m} \mathrm{Al} \mathrm{Al}_{2} \mathrm{O}_{3}$ thin film, measured at $30 \mathrm{keV}$. The sample surface was tilted by different angles $\psi$ relative to the scattering plane. The asymmetry in the peaks at different tilt angles can be used to determine the stress depth profile shown in the bottom panel.

stress was measured in a $6 \mu \mathrm{m} \mathrm{Al}_{2} \mathrm{O}_{3}$ thin-film coating on a cemented WC substrate. The surface normal was tilted by an angle $\psi$ with respect to the scattering plane to determine the residual stress at different angles to the surface. The upper part of Fig. 6 shows the (116) peak profile for three different values of $\psi$. At $\psi=0^{\circ}$ the profile shows a marked asymmetry which disappears at $\psi=42.13^{\circ}$ and is reversed at $\psi=67.215^{\circ}$. This asymmetry is caused by a steep stress depth profile, which is not easy to resolve vertically in a thin film (Genzel, 1997), but can here be determined from the data and is shown in the lower part of Fig. 6. The disappearance of the asymmetry at $42.13^{\circ}$ shows that in this direction there is no lattice distortion due to stress.

\section{Summary}

The new beamline MAGS at BESSY provides a focused hard $\mathrm{X}$-ray beam with high fluxes that can be used in a wide range of diffraction experiments. Its flexible optics allow rapid and reproducible switching between different beamline configurations. So far the beamline optics exposed to the white beam have coped well with the high heat load from the wiggler source. The experimental set-up can be used for experiments in many different scattering geometries in a wide sample temperature range from 4 to $800 \mathrm{~K}$, and with different detector configurations.

The building of this beamline has been supported by the BMBF through the HGF Vernetzungsfonds, grant Nos. 01SF0005 and 01SF0006. We also wish to thank the BESSY staff for their support, particularly Dr Erko and Dr Schäfers.

\section{References}

Berger, D., Weihreter, E., Mezentsev, N. \& Shkaruba, V. (2002). 8th European Particle Accelerator Conference (EPAC 2002), Paris, France, 3-7 June 2002, pp. 2595-2597. Geneva: European Physical Society.

Blume, M. \& Gibbs, D. (1988). Phys. Rev. B, 37, 1779-1789.

Dudzik, E., Feyerherm, R., Waterstradt, T., Schröder, L.-E., Diete, W. \& Frahm, R. (2006). To be published.

Feyerherm, R., Dudzik, E., Aliouane, N. \& Argyriou, D. (2006). Phys. Rev. B, 73, 180401(R).

Freund, A. K., Comin, F., Hazemann, J.-L., Hustache, R., Jenninger, B., Lieb, K. \& Pierre, M. (1998). Proc. SPIE, 3448, 144-155.

Genzel, Ch. (1997). Phys. Status Solidi A, 159, 283-296.

Genzel, Ch., Denks, I. A. \& Klaus, M. (2006). Proceedings of the Seventh European Conference on Residual Stress (ECRS 7). To be published.

Hill, J. P. \& McMorrow, D. F. (1996). Acta Cryst. A52, 236-244.

Meß, K. H., Tröger, L. \& Brüggmann, U. (1998). HASYLAB Annual Report. HASYLAB, Hamburg, Germany.

Swislow, G. (1996). spec. Certified Scientific Software, Cambridge, MA, USA.

Zaeper, R., Richwin, M., Lützenkirchen-Hecht, D. \& Frahm, R. (2002). Rev. Sci. Instrum. 73, 1564-1567. 